\title{
ACHIEVABLE TRANSVERSE CYLINDRICAL ELECTROMAGNETIC MODE
}

\author{
R. CHEN(1) AND X. LI(2)
}

\begin{abstract}
The system of Maxwell equations with an initial condition in a vacuum is solved in a cylindrical coordinate system. It derives the cylindrical transverse electromagnetic wave mode in which the electric field and magnetic field are not in phase. Such electromagnetic wave can generate and exist in actual application, and there is no violation of the law of conservation of energy during the electromagnetic field interchanges.
\end{abstract}

\section{INTRODUCTION}

The general solutions of Maxwell equations [1, 2] contain unnumbered forms of wave modes in both magnetic field and electric field, but only the special solutions that obey its initial or boundary conditions can describe the achievable electromagnetic wave.

However, classical theories discuss time-harmonic transverse electromagnetic waves without verifying whether initial conditions are achieved, but usually predefine the forms of the solutions to the Maxwell equations [3, 4, 5, 6] by adopting the plural expressions, $\mathbf{E}(\mathbf{r}, t)=\mathbf{E}(\mathbf{r}) e^{i \omega t}$ and $\mathbf{B}(\mathbf{r}, t)=\mathbf{B}(\mathbf{r}) e^{i \omega t}$. Consequently, the electric field and magnetic field of the time-harmonic electromagnetic wave obtained above are in-phase everywhere. These electromagnetic modes may form an initial-moment crisis and violate the conservation of energy during the electric and magnetic fields interchanges to each other. We note that the acknowledged results achieved by some previous authors [7] are actually derived from the above predefined plural expressions of the solutions of the Maxwell equations. In classical theory of electromagnetic wave, the substantial existence of electromagnetic waves is given little attention to [8], because both the propagation and reception of electromagnetic waves are independent of their respective phase differences actually. There were no immediate details about whether one can design and run an experiment to test if it exists for the electromagnetic wave in which the electronic field and magnetic field in phase. However, a physical theory must always guarantee the consistency of its mathematical deductions. This is just why we restudy the problem of initial value for the Maxwell equations. According to the theory of differential equations, solutions of Maxwell equations should not violate natural initial and boundary conditions [9, 10]. Even if an excited source and the consequent radiation of the electromagnetic waves change according to pure oscillations by the same frequency, the generated electric fields and magnetic fields cannot be in same phase with each other, otherwise the energy conservation law would be violated.

We solved the problem of initial value for the plane transverse electromagnetic mode 11. It shows that the classical plane electromagnetic wave may be unable to produce practically, but this conclusion does not have an effect on the general application of 
electromagnetic waves [12, 13, 14, 15, 16. Unlike the classical plane electromagnetic wave modes, if the time harmonic wave propagates along the positive direction of $\mathrm{x}$-axis, the solutions of the Maxwell equations that accord with an initial value condition in a vacuum would be the following forms [1]

or

$$
\begin{aligned}
& \mathbf{E}=\mathbf{e}_{y} E_{m} \sin \omega\left(t-\frac{x}{c}\right) \\
& \mathbf{B}=\mathbf{e}_{z} \frac{E_{m}}{c}\left[\sin \frac{\omega x}{c}+\sin \omega\left(t-\frac{x}{c}\right)\right]
\end{aligned}
$$

$$
\begin{aligned}
& \mathbf{E}=\mathbf{e}_{y} E_{m} \cos \omega\left(t-\frac{x}{c}\right) \\
& \mathbf{B}=\mathbf{e}_{z} \frac{E_{m}}{c}\left[\cos \omega\left(t-\frac{x}{c}\right)-\cos \frac{\omega x}{c}\right]
\end{aligned}
$$

the wave modes obtained above meet with explicit physical meanings.

Here we follow the work that derives the equations (11) and (2) to discuss the problem of initial value for the cylindrical transverse electromagnetic mode. We obtain the solutions of Maxwell equations with a sort of initial condition in a cylindrical coordinate system. Its electric field and magnetic field are of axial symmetry, with electric field vectors pointing to the radical direction of the coaxial cylinder and magnetic field vectors to the tangential direction. It is similar to the conclusion upon the plane transverse electromagnetic mode, which is practically able to produce and exist. That is, the magnetic field and electric field of the cylindrical transverse electromagnetic wave are in different phase, and their amplitude ratio depends on the coordinate of space as well.

\section{Initial CONDitions of time-HaRmonic Cylindrical Transverse Waves}

In solving simultaneous differential equations, definite conditions, including initial conditions and boundary conditions, are required to be determined [17]. If the space is infinite, then we only need to consider the initial condition. In fact, since the initial moment can be selected at random, the initial condition of the Maxwell equations does exist in any situation. The coaxial transmission of electromagnetic waves in hollow conductors and core wires is presented in Figure 1.1. The radius of the wire is $R_{1}$, and that of the hollow conductor is $R_{2}$. In a vacuum, if the wire and the hollow conductor are having the same amount of positive and negative charge (the electric quantity per unit length along the axes on surface is $\lambda_{m}$ ), then the electrostatic field inside the conductor can be written as

$$
\mathbf{E}=\frac{\lambda_{m}}{2 \pi \varepsilon_{0} r} \mathbf{e}_{r}
$$

where $R_{1} \leq r \leq R_{2}$. Among which $\mathbf{e}_{r}$ is radical unit vector, and $r$ is the distance from a certain point in the coaxial transmission line to the axis.

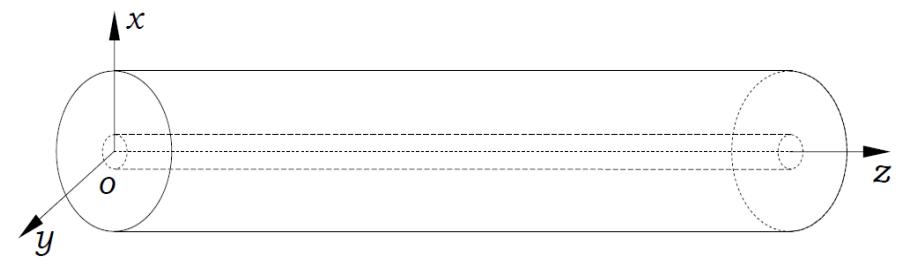

Figure 1. Cylindrical transverse electromagnetic wave inside the coaxial transmission line 
When the left side of the conductor is connected to a periodically changing power supply, the distribution of charges thus varies and propagates towards right, which forms electric field waves transmitting along the positive direction of $\mathrm{z}$, vertical to the direction of the electric field. If we have the apart of the hollow conductor with the wire very close, and the electric field steadily distributed, then, based on the distribution formula of electrostatic field (3), the formula of the electric field wave in a vacuum can also be determined.

$$
\begin{aligned}
& \mathbf{E}=\mathbf{e}_{r} \frac{\lambda_{m}}{2 \pi \varepsilon_{0} r} f(z, t) \\
& E(t=0)=0 \\
& E(z=0)=\mathbf{e}_{r} \frac{\lambda_{m}}{2 \pi \varepsilon_{0} r} f(t) \\
& \mathbf{B}(t=0)=0
\end{aligned}
$$

where $f(z, t)$ may be simple harmonic, which is determined by the charge distribution on the surface of conductor. If the excitation sources is an alternating currents of high frequency, which the oscillation current changes according sine function, then the corresponding wave mode with the initial and boundary conditions in a vacuum will be expressed by plural expressions

$$
\begin{aligned}
& \mathbf{E}=\mathbf{e}_{r} \frac{\lambda_{m}}{2 \pi \varepsilon_{0} r} e^{i \omega\left(t-\frac{z}{c}\right)} \\
& E(t=0)=0 \\
& E(z=0)=\mathbf{e}_{r} \frac{\lambda_{m}}{2 \pi \varepsilon_{0} r} e^{i \omega t} \\
& \mathbf{B}(t=0)=0
\end{aligned}
$$

At the initial moment, the electric field within the transmission wire is zero, and hence the magnetic field is zero as well, which build up the initial conditions.

\section{General solutions of Maxwell equations in Cylindrical Coordinate SYSTEM}

Periodically changing electric fields generate periodically changing magnetic fields, and the correspondingly generated electromagnetic wave propagates at the speed of light in vacuum. The expressions of the electric field and the magnetic field satisfy Maxwell equations

$$
\begin{aligned}
& \nabla \times \mathbf{E}=-\frac{\partial \mathbf{B}}{\partial t} \\
& \nabla \times \mathbf{B}=\frac{1}{c^{2}} \frac{\partial \mathbf{E}}{\partial t} \\
& \nabla \cdot \mathbf{E}=0 \\
& \nabla \cdot \mathbf{B}=0
\end{aligned}
$$


Since the problem is axially symmetric, in cylindrical coordinate system [18], the simultaneous equation (6) can be written as [19]

$$
\begin{aligned}
& \mathbf{e}_{r}\left(\frac{1}{r} \frac{\partial E_{z}}{\partial \theta}-\frac{\partial E_{\theta}}{\partial z}\right)+\mathbf{e}_{z}\left[\frac{1}{r} \frac{\partial}{\partial r}\left(r E_{\theta}\right)-\frac{1}{r} \frac{\partial E_{r}}{\partial \theta}\right]+\mathbf{e}_{\theta}\left(\frac{\partial E_{r}}{\partial z}-\frac{\partial E_{z}}{\partial r}\right) \\
& =-\frac{\partial \mathbf{B}}{\partial t} \\
& \mathbf{e}_{r}\left(\frac{1}{r} \frac{\partial B_{z}}{\partial \theta}-\frac{\partial B_{\theta}}{\partial z}\right)+\mathbf{e}_{z}\left[\frac{1}{r} \frac{\partial}{\partial r}\left(r B_{\theta}\right)-\frac{1}{r} \frac{\partial B_{r}}{\partial \theta}\right]+\mathbf{e}_{\theta}\left(\frac{\partial B_{r}}{\partial z}-\frac{\partial B_{z}}{\partial r}\right) \\
& =\frac{1}{c^{2}} \frac{\partial \mathbf{E}}{\partial t} \\
& \frac{1}{r} \frac{\partial}{\partial r}\left(r E_{r}\right)+\frac{1}{r} \frac{\partial E_{\theta}}{\partial \theta}+\frac{\partial E_{z}}{\partial \tilde{B}_{z}}=0 \\
& \frac{1}{r} \frac{\partial}{\partial r}\left(r B_{r}\right)+\frac{1}{r} \frac{\partial B_{\theta}}{\partial \theta}+\frac{\partial{ }^{2}}{\partial z}=0
\end{aligned}
$$

As to electric mode (4), since $E_{r}=E_{r}(r, z, t), E_{\theta}=0 a n d E_{z}=0$, simultaneous equations (7) can be rewritten as

$$
\begin{aligned}
& \mathbf{e}_{\theta} \frac{\partial E_{r}}{\partial z}=-\mathbf{e}_{r} \frac{\partial B_{r}}{\partial t}-\mathbf{e}_{\theta} \frac{\partial B_{\theta}}{\partial t}-\mathbf{e}_{z} \frac{\partial B_{z}}{\partial t} \\
& \mathbf{e}_{r}\left(\frac{1}{r} \frac{\partial B_{z}}{\partial \theta}-\frac{\partial B_{\theta}}{\partial z}\right)+\mathbf{e}_{z}\left[\frac{1}{r} \frac{\partial}{\partial r}\left(r B_{\theta}\right)-\frac{1}{r} \frac{\partial B_{r}}{\partial \theta}\right]+\mathbf{e}_{\theta}\left(\frac{\partial B_{r}}{\partial z}-\frac{\partial B_{z}}{\partial r}\right) \\
& =\mathbf{e}_{r} \frac{1}{c^{2}} \frac{\partial E_{r}}{\partial t} \\
& \frac{1}{r} \frac{\partial}{\partial r}\left(r E_{r}\right)=0 \\
& \frac{1}{r} \frac{\partial}{\partial r}\left(r B_{r}\right)+\frac{1}{r} \frac{\partial B_{\theta}}{\partial \theta}+\frac{\partial B_{z}}{\partial z}=0
\end{aligned}
$$

According to the third equation above, the electric field satisfies the equation $r E_{r}=$ $g(z, t)$, so that

$$
E_{r}=\frac{g(z, t)}{r}
$$

The result is in accordance with that deduced by (44), which means the third equation of (8) is valid. Because that both sides of the equation are correspondingly equal to each other, so that the other three equations can be transformed into

$$
\begin{aligned}
& \frac{\partial B_{r}}{\partial t}=0 ; \quad \frac{\partial B_{z}}{\partial t}=0 ; \quad \frac{\partial B_{\theta}}{\partial t}=-\frac{\partial E_{r}}{\partial z} \\
& \frac{1}{r} \frac{\partial B_{z}}{\partial \theta}-\frac{\partial B_{\theta}}{\partial z}=\frac{1}{c^{2}} \frac{\partial E_{r}}{\partial t} ; \quad \frac{\partial B_{r}}{\partial z}-\frac{\partial B_{z}}{\partial r}=0 \\
& \frac{1}{r} \frac{\partial}{\partial r}\left(r B_{\theta}\right)-\frac{1}{r} \frac{\partial B_{r}}{\partial \theta}=0 \\
& \frac{1}{r} \frac{\partial}{\partial r}\left(r B_{r}\right)+\frac{1}{r} \frac{\partial B_{\theta}}{\partial \theta}+\frac{\partial B_{z}}{\partial z}=0
\end{aligned}
$$

According to the first three equations above, we get

$$
\begin{aligned}
& B_{r}=B_{r}(r, \theta, z) \\
& B_{z}=B_{z}(r, \theta, z) \\
& B_{\theta}=-\int \frac{\partial E_{r}}{\partial z} d t+a(r, \theta, z)
\end{aligned}
$$


This result will certainly satisfy the last two equations of (10). Consequently, the magnetic field can be written as

$$
\mathbf{B}=\mathbf{e}_{r} B_{r}(r, \theta, z)+\mathbf{e}_{\theta}\left[-\int \frac{\partial E_{r}}{\partial z} d t+a(r, \theta, z)\right]+\mathbf{e}_{z} B_{z}(r, \theta, z)
$$

By using the initial condition $\mathbf{B}(t=0)=0$, we get

$$
B_{r}(r, \theta, z)=0 ; \quad B_{z}(r, \theta, z)=0
$$

Therefore,

$$
\mathbf{B}=\mathbf{e}_{\theta}\left[-\int \frac{\partial E_{r}}{\partial z} d t+a(r, \theta, z)\right]
$$

On the other hand, by the use of (13), and the integration of the first equation on the second line of (10), we get

$$
\mathbf{B}=\mathbf{e}_{\theta}\left[-\int \frac{1}{c^{2}} \frac{\partial E_{r}}{\partial t} d z+b(r, \theta, t)\right]
$$

Based on (4) and (14), the general equation of cylindrical electromagnetic mode can be written as

$$
\begin{aligned}
& \mathbf{E}=\mathbf{e}_{r} \frac{\lambda_{m}}{2 \pi \varepsilon_{0} r} f(z, t) \\
& \mathbf{B}=\mathbf{e}_{\theta}\left[-\int \frac{\partial E_{r}}{\partial z} d t+a(r, \theta, z)\right] \\
& \mathbf{B}(t=0)=0
\end{aligned}
$$

or

$$
\begin{aligned}
& \mathbf{E}=\mathbf{e}_{r} \frac{\lambda_{m}}{2 \pi \varepsilon_{0} r} f(z, t) \\
& \mathbf{B}=\mathbf{e}_{\theta}\left[-\int \frac{1}{c^{2}} \frac{\partial E_{r}}{\partial t} d z+b(r, \theta, t)\right] \\
& \mathbf{B}(t=0)=0
\end{aligned}
$$

(16) and (17) are equivalent to each other. In a vacuum, they are both general solutions of the Maxwell equations, where the magnetic field provoked by a radical electric field.

\section{Time-harmonic Cylindrical Transverse Electromagnetic Mode}

On the basis of (16) or (17), the expression of magnetic field can be acquired by knowing the expression of electric field. By inserting (5) into (16), we get

$$
\begin{aligned}
& \mathbf{E}=\mathbf{e}_{r} \frac{\lambda_{m}}{2 \pi \varepsilon_{0} r} e^{i \omega\left(t-\frac{z}{c}\right)} \\
& \mathbf{B}=\mathbf{e}_{\theta}\left[-\int \frac{\partial E_{r}}{\partial z} d t+a(r, \theta, z)\right] \\
& \mathbf{B}(t=0)=0
\end{aligned}
$$

According to the first equation, we get

$$
\frac{\partial E_{r}}{\partial z}=-\frac{i \omega}{c} \frac{\lambda_{m}}{2 \pi \varepsilon_{0} r} e^{i \omega\left(t-\frac{z}{c}\right)}
$$

By inserting (19) into the second equation in (18), and then integrating it, we get

$$
\mathbf{B}=\mathbf{e}_{\theta}\left[\frac{1}{c} \frac{\lambda_{m}}{2 \pi \varepsilon_{0} r} e^{i \omega\left(t-\frac{z}{c}\right)}+a(r, \theta, z)\right]
$$

Using the initial conditions of (18), the undetermined function can be confirmed

$$
a(r, \theta, z)=-\frac{1}{c} \frac{\lambda_{m}}{2 \pi \varepsilon_{0} r} \mathrm{e}^{-i \omega \frac{z}{c}}
$$


After substituting the function into (20), we further obtain

$$
\mathbf{B}=\mathbf{e}_{\theta} \frac{\lambda_{m}}{2 \pi c \varepsilon_{0} r}\left[e^{i \omega\left(t-\frac{z}{c}\right)}-e^{-i \omega \frac{z}{c}}\right]
$$

Therefore, (18) can be transformed into

$$
\begin{aligned}
& \mathbf{E}=\mathbf{e}_{r} \frac{\lambda_{m}}{2 \pi \varepsilon_{0} r} e^{i \omega\left(t-\frac{z}{c}\right)} \\
& \mathbf{B}=\mathbf{e}_{\theta} \frac{\lambda_{m}}{2 \pi c \varepsilon_{0} r}\left[e^{i \omega\left(t-\frac{z}{c}\right)}-e^{-i \omega \frac{z}{c}}\right]
\end{aligned}
$$

So we have the expressions of electromagnetic waves (23) in a vacuum satisfying every single component equation of Maxwell (10), and hence satisfying Maxwell equations. The expression (23) is a particular solution of Maxwell's equations that accord with initial conditions. It represents a kind of existing transverse cylindrical time-harmonic electromagnetic wave mode. We obtain the electromagnetic wave mode generated by sinusoidal electric wave by using the imaginary part of (23).

$$
\begin{aligned}
& \mathbf{E}=\mathbf{e}_{r} \frac{\lambda_{m}}{2 \pi \varepsilon_{0} r} \sin \omega\left(t-\frac{z}{c}\right) \\
& \mathbf{B}=\mathbf{e}_{\theta} \frac{\lambda_{m}}{2 \pi c \varepsilon_{0} r}\left[\sin \omega\left(t-\frac{z}{c}\right)+\sin \frac{\omega z}{c}\right]
\end{aligned}
$$

By inserting the real part of (23), the electromagnetic wave generated by cosine electric wave can be obtained

$$
\begin{aligned}
& \mathbf{E}=\mathbf{e}_{r} \frac{\lambda_{m}}{2 \pi \varepsilon_{0} r} \cos \omega\left(t-\frac{z}{c}\right) \\
& \mathbf{B}=\mathbf{e}_{\theta} \frac{\omega \lambda_{m}}{2 \pi c \varepsilon_{0} r}\left[\cos \omega\left(t-\frac{z}{c}\right)-\cos \frac{\omega z}{c}\right]
\end{aligned}
$$

\section{Conclusions}

From Maxwell' theory, if the electric field in space changes, the magnetic field at the same place would change and these changing electric field and magnetic field will generate new changing electric field and magnetic field in farther space. Consequently, the changed electric field and magnetic field are not confined to a region but propagate from near site to farther places. The propagation of electromagnetic field forms electromagnetic waves. The characteristics of the electromagnetic wave are actually described by the solutions [20, 21, 22] of the Maxwell equations. The electromagnetic wave is a transverse wave, which is just determined by the transverse wave conditions of the Maxwell equations, $\nabla \cdot \mathbf{E}=0$ and $\nabla \cdot \mathbf{B}=0$.

Since the classical theory first preconcerts the forms of solutions before solving the Maxwell equations, the obtained transverse electromagnetic wave mode shows a most incredible characteristic that the electric field and magnetic field are in phase everywhere. Now that the periodic changing electric field generate periodic changing magnetic field, the process of the transmission of an electromagnetic wave is that of energy radiation, and the electromagnetic energy is transmitted with the electromagnetic waves. If the maximum or minimum value of electric field and magnetic field in an electromagnetic wave are always obtained simultaneously, how do the electric energy and magnetic energy interchange? Consequently, we conclude that the classical electromagnetic wave in which the electric field and magnetic field are in phase everywhere violates the law of conservation of energy.

We strive to maintain the conclusions of classical theory, but the initial condition of Maxwell equations has always been there. We wish to know the solutions of the Maxwell 
equations with a certain initial condition. The cylindrical transverse electromagnetic wave modes in a vacuum are hence derived. It can be determined that, with the solutions of Maxwell equations obtained under initial values, the existing electric field vector and magnetic field vector, the velocity vector of the plane or cylindrical electromagnetic wave are vertical to one another. Nevertheless, the amplitude ratio of electric field and magnetic field is not constant in space, and their phase difference changes as well, so that they do not reach maximum or zero simultaneously.

\section{REFERENCES}

[1] Maxwell, J. C., "A dynamical theory of the electromagnetic field," Phil. Trans. Roy. Soc. London, Vol. 155, 459-512, 1865.

[2] Van Bladel, J., Electromagnetic Fields, McGraw-Hill, New York, 1964.

[3] Jackson, J. D., Classical Electrodynamics, 3rd Edition, John Wiley and Sons, 1998.

[4] Landau, L. D. and E. M. Lifshitz, The classical theory of fields, Butterworth-Heinemann, 1980.

[5] Tsang, T., Classical electrodynamics, World Scientific Pub. Co. Inc., 1997.

[6] Yang, R. R. G. and T. T. Y. Wong, Electromagnetic fields and waves, Higher Education Press, 2006.

[7] Harrington, R. F., Time-Harmonic Electromagnetic Fields, McGraw-Hill, New York, NY, 1961.

[8] Brittingham, J. N., "Focus waves modes in homogeneous Maxwell equations: Transverse electric mode," J. Appl. Phys. Vol. 54, 1179-1189, 2009.

[9] Chen, R., "The Optimum Differential Equations," Chin. J. Engin. Math. Vol. 17, 82-86, 2000.

[10] Chen, R., "The Uniqueness of the Eigenvalue Assemblage for Optimum Differential Equations," Chin. J. Engin. Math., Vol. 20, 121-124, 2003.

[11] Chen, R., "The Problem of Initial Value for the Plane Transverse Electromagnetic Mode," Acta Phys. Sin-CH ED, Vol. 49, 2514-2518, 2000.

[12] Coleman, S. and D. Grischkowsky, "A THz transverse electromagnetic mode two-dimensional interconnect layer incorporating quasi-optics," Appl. Phys. Lett., Vol. 83, 3656-3658, 2003.

[13] Chu, C. and T. Ohkawa, "Transverse Electromagnetic Waves," Phys. Rev. Lett., Vol. 48, 837-838, 1982.

[14] Davis, L. W. and G. Patsakos, "TM and TE electromagnetic beams in free space," Opt. Lett, Vol. $6,22-23,1981$.

[15] Chew, W. C. and W. H. Weedon, "A 3D perfectly matched medium from modified Maxwell's equations with stretched coordinates," Micro. opt. tech. lett., Vol. 7, 599-604, 1994.

[16] Elliott, R. S., Electromagnetics-History, Theory, and Applications, IEEE Press New York, 1993.

[17] Karlsson, A. and G. Kristensson, "Constitutive relations, dissipation, and reciprocity for the Maxwell equations in the time domain," J. of Electromagn. Waves and Appl., Vol. 6, 537-551, 1992.

[18] Ruan, Z., M. Yan, C. W. Neff, and M. Qiu, "Ideal cylindrical cloak: perfect but sensitive to tiny perturbations," Phys. Rev. Lett., Vol. 99, 113903, 2007.

[19] Isic, G., Gajic, R., Novakovic, B., Z. Popovic, and K. Hingerl, "Radiation and scattering from imperfect cylindrical electromagnetic cloaks," Opt. Express, Vol. 16, 1413-1422, 2008.

[20] Naqvi, A., S. Ahmed, and Q. A. Naqvi, "Perfect electromagnetic conductor and fractional dual interface placed in a chiral nihility medium." J. of Electromagn. Waves and Appl., Vol. 24, 19911999, 2010.

[21] Chen, H. Y., L. J. Deng, and P. H. Zhou. "Suppression of surface wave from finite conducting surfaces with impedance loading at margins." J. of Electromagn. Waves and Appl., 24, 1977-1989, 2010.

[22] Pinel, N., J. Saillard, and C. Bourlier, "Extension of the Roughness Criterion of a One-step Surface to a One-Step Layer." J. of Electromagn. Waves and Appl., 24, 1195-1205, 2010.

(1)Shenzhen Institute of Physics and Mathematics, Shenzhen, 518028, China,

(2)South China Normal University, Guangzhou, 510631, China, 